\title{
Early diagenesis of carbohydrates and lignin in mangrove sediments subject to variable redox conditions (French Guiana)
}

\author{
C. Marchandạ, J .R. Disnarạ, E. Lallier-Vergèsạ and N. Lottierẹ \\ alnstitut des Sciences de la Terre d'O réans, UMR 6113 CNRS-UO, Université \\ d'Orléans, Géosciences, 45067 Orléans, France
}

\section{Abstract}

A comparative study of lignin and neutral carbohydrate compositions, combined with $\mathrm{C}, \mathrm{N}$ and $\delta^{13} \mathrm{C}$ analyses, was carried out on sedimentary cores, and on various vascular plant species collected in mangrove swamps of French Guiana. The main purpose of this study was to assess the diagenesis of carbohydrates and lignin in brackish to hypersaline fine-grained mangrove sediments characterized by great changes in redox conditions. Distribution of carbohydrates in sediments reflects both the lability of these compounds and their efficient recycling. They are subject to selective degradation, cellulosic glucose and xylose appearing to be the two most labile neutral sugars. In contrast a relative increase in arabinose, rhamnose, fucose and hemicellulosic glucose between plants and sediments, suggests that they may be more refractory and/or that they also derive from microbial synthesis. The total carbon from lignin-derived phenols is higher in sediments than in mangrove plants as a consequence of their rather refractory character. Nevertheless, evidence of lignin decomposition was found to be independent of local environmental conditions. The various redox processes that occur in mangrove sediments depend on plant species, stages in forest development and season. Different redox conditions induce different mechanisms for the decomposition of lignin and thus induce changes in phenol distributions. At depth, in most mangroves, an increase in (Ad/Al) $v$ ratios and in deoxy sugars (fucose and rhamnose) content was significantly correlated with increased proportions of oxidized allochthonous organic debris deriving from the Amazonian detrital discharge, thus suggesting a specific source effect rather than a diagenesis induced change. Therefore, this study illustrates that both lignin and cellulose, derived from vascular plant debris, can be degraded in waterlogged mangrove sediments, and that their distribution depends on environmental conditions.

\section{Introduction}

Detritus from vascular plants represent an important contribution to the organic matter in the marine environment. Every year, n 0.4 Gt. of organic 
carbon are carried to coastal environments representing $0.7 \%$ of the terrestrial primary production (Schlesinger and Melack, 1981; Meybeck, 1982; Ittekkot, 1988). Wetlands deliver $\sim 15 \%$ of this flux (Schlesinger and Melack, 1981). The particulate input of terrestrial organic matter (TOM) to the marine environment largely exceeds the total burial rate of total organic carbon in sediments, consequently this TOM is submitted to extensive and rapid remineralisation processes (Prahl et al., 1994; Hedges et al., 1997).

During the last decades, polysaccharides (mainly cellulose and hemicelluloses) and lignin have been widely investigated to identify the sources and the fate of organic matter in the open ocean (Hedges and Parker, 1976; Hedges and Mann, 1979b; Cowie and Hedges, 1984; Ertel and Hedges, 1985; Bianchi et al., 1999; Kerhervé et al., 2002). However, few studies were interested in comparing their fates in marine sediments as well as their complementarity as sources and diagenetic indicators (Hedges et al., 1985; Benner et al., 1990; Cowie et al., 1992).

Carbohydrates represent the largest fraction of photosynthetically assimilated carbon in the biosphere (Cowie and Hedges, 1984). They are the most abundant constituents of vascular plants where they mostly occur in cell wall material and play a major role as storage and structural components. Considering their diversity ( $\approx 200$ polysaccharides occur naturally; Greenland and Oades, 1975), and their ubiquity (they are present in vascular plants, in marine plankton and in bacteria), polysaccharides are potential indicators of OM sources. However, bacteria and fungi rapidly degrade polysaccharides and synthesize new ones.

Lignin is a nitrogen free co-polymer of various phenyl-propenyl alcohols that is abundant in vascular plants. It is present in cell walls where it imposes rigidity and minimizes water permeation. Lignin compositions vary significantly between gymnosperms and angiosperms, and between hard (i.e., woods) and soft (i.e., leaves, needles) tissues. Because of these compositional differences, an exclusive association with higher plants, and a well-known resistance to microbial degradation (Sarkanen and Ludwig, 1971), lignin is usually considered as a more specific tracer of land plant remains than others indicators (e.g., $\mathrm{C} / \mathrm{N}$ ratios and $\delta^{13} \mathrm{C}$ ) and is thus, frequently used as such. Upon cupric oxide oxidation, lignin yields eight dominant phenolic compounds, which reflect the amounts and sources of parent polymer (Hedges and Mann, 1979a; Hedges and Ertel, 1982). In addition, some specific monomeric ratios may be used as indicators of lignin diagenetic alteration (Ertel and Hedges, 1984; Hedges et al., 1988).

Mangrove forests are specific wetlands, developing in the intertidal zone, that have a high adaptation capacity to extreme environmental conditions (from fresh to hypersaline waters, waterlogging, contrasting redox conditions). Considering their widespread distribution along the tropical and subtropical coastline where they cover ca. $1810^{6}$ ha (Spalding et al., 1997), mangroves 
play an important role in the global carbon cycle. They are characterized by a high net primary production of organic matter (Odum and Heald, 1975; Clough, 1992), intense turnover (Alongi et al., 1999) and burial. In French Guiana, mangroves develop on sediments coming from the huge particulate discharge of the Amazon River, partly deflected northwestwards by the current of the Guianas and then settling in a series of mudwaves migrating towards the Orinoco River. The dynamic aspect of this coastline results in limited mangrove lifetime, i.e., 30 to 50 years. In a previous study concerning the bulk characterization of organic matter in this environment (Marchand ef al., 2003), the organic content appeared to be a blend of strongly oxidized debris deriving from Amazon River detritus and $\mathrm{OM}$ deriving from algal mats and vascular plants, which are introduced in the upper $30 \mathrm{~cm}$. Below this depth, sediments characteristics are those of the shoreface one, i.e., mainly composed of oxidized debris. In the present work, our first intent was to get a more refined interpretation of organic matter sources and diagenesis through major biopolymer analysis than that obtained solely from bulk characterization. More specifically, our purpose was to assess the effect of diagenesis on the distribution of carbohydrates and lignin derived phenols in marine sediments characterized by great modifications of the redox conditions, the latter depending on plant species, seasons, amount and reactivity of organic matter. We here report on a comparative study of lignin and carbohydrate composition combined with elemental (Carbon and Nitrogen) and stable isotopic $\left(\delta^{13} \mathrm{C}\right)$ data. Qualitative and quantitative analyses were carried out on woody and herbaceous tissues from various vascular plant species specific to that environment, and on samples from sedimentary cores collected in 5 mangroves areas differentiated following their species composition and stage of evolution.

\section{Materials and methods}

\subsection{Study site}

The studied mangroves are located: i) on the right bank of the Sinnamary River, $50 \mathrm{~km}$ northwest of Kourou, ii) at the mouth of the Kaw River, $30 \mathrm{~km}$ southeast of Cayenne, French Guiana (Fig. 1). These mangroves develop on the upper intertidal zone of huge $30 \mathrm{~km}$ long mud banks that can reach up to $5 \mathrm{~km}$ in width. Sinnamary mangroves are Avicennia geminans dominated. However scarce Laguncularia racemosa and Crenea maritima stands colonized the seaward fringe of mangrove swamps. Some Acrostichum aureum developed in the senescent mangrove. 


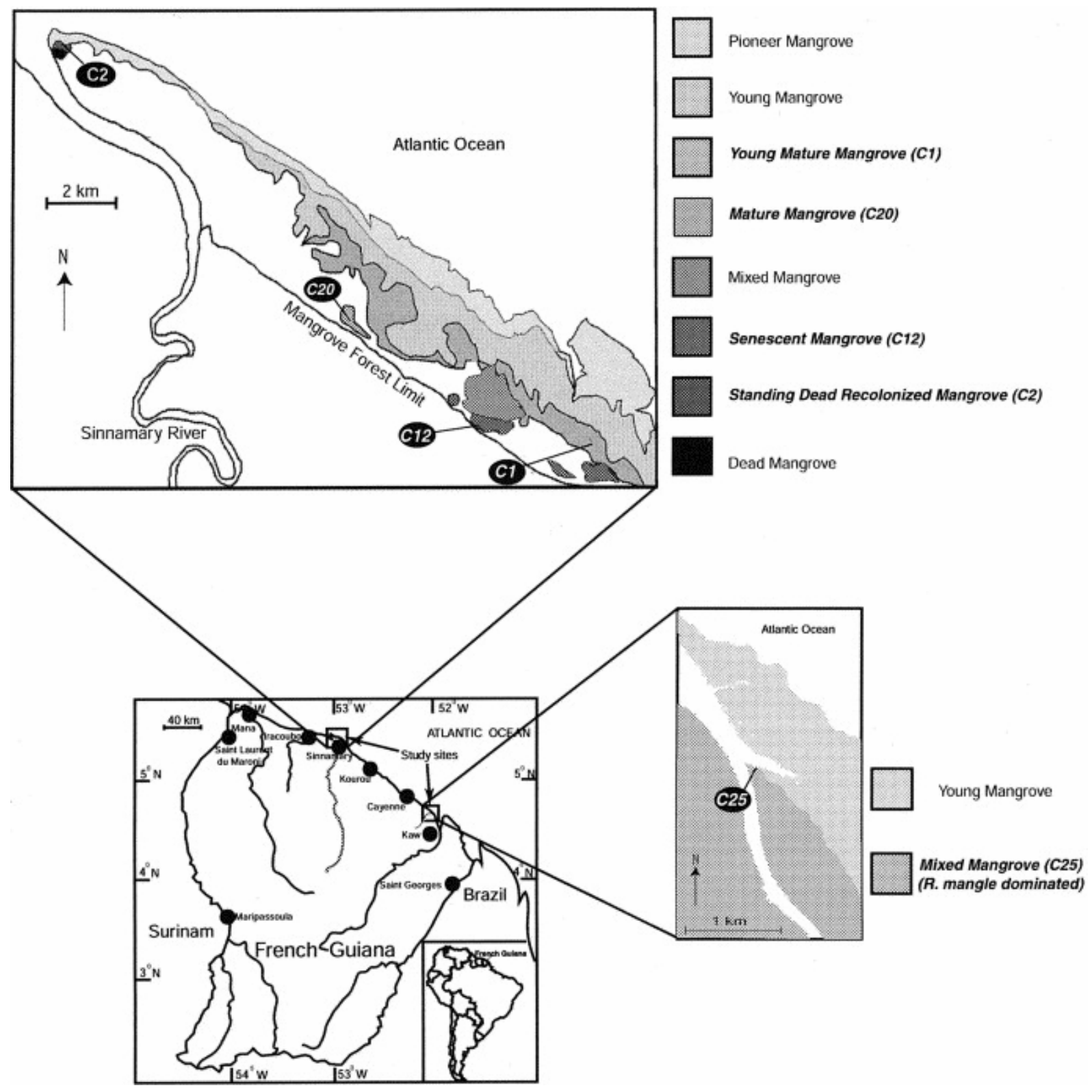

Fig. 1. Map of the studied area showing the localisation of cores (adapted from Fromard et al., 1998).

In Kaw, sediment cores were performed beneath Rhizophora mangle stands. Rhizophora mangroves have a fibrous nature and abundant rootlets while black Avicennia mangroves have a radial cable root system developing close to sediment surface (Hesse, 1961). Avicennia geminans, Laguncularia racemosa, Rhizophora mangle and Crenea maritima are dicotyledonous angiosperms having a C3 photosynthesis and being considered as halophytes. Acrostichum aureum is a fern having C3 photosynthesis. In the early stages of mangrove evolution, different types of algal-microbial mats cover the sediment: diatoms, cyanophyceae, and micro-green algae (De Wit, oral communication). The frequency of biofilms decreases rapidly with forest age. 
The higher topographic level of the mature mangrove zone prevents all but spring tides to overflow them and so to reach the soil of the mature and senescent mangrove zones. Consequently, tidal flushing is limited and leaf litter accumulates in the senescent mangrove zone. The various developmental stages of the Avic ennia-dominated mangrove of Sinnamary were described relative to their structural and biologic features (Fromard ef al., 1998). Due to the dynamic features of the coastline, mangroves trees sometimes died from a sudden accretion of sediments that bury the pneumatophores. This process induced areas of dead mangroves that can be further colonized, resulting in the so-called "recolonized cemetery forest."

\subsection{Field sampling}

Cores were collected at low tide with an Eijkelkamp gouge auger, in 5 areas defined in terms of specific composition and stages of development of the vegetation. Work was carried out in two fieldtrips, in July 2001 and January 2002. In the A. germinans forests, core locations were chosen equidistant from trees, in consideration of the large extension of the radial cable roots. Concerning the R. mangle forests, cores were collected under prop roots. After being collected, cores were wrapped in plastic film and aluminum foil to limit gaseous exchanges. Cores were sampled on the day of coring, and samples were kept refrigerated until analysis. Organic tissues (fresh leaves and wood) were sampled on trees surrounding the mud coring.

\subsection{Carbon and nitrogen}

Elemental composition (carbon and nitrogen) was determined by combustion at $1100^{\circ} \mathrm{C}$ with a CNS-2000 LECO apparatus. Due to the nearly total lack of carbonates (samples were tested with $\mathrm{HCl}$ ), total carbon is assumed to be total organic carbon (TOC). Samples were taken at the following depth-intervals (in cm): $2 \mathrm{~cm}$ from 0 to $20 \mathrm{~cm}, 5 \mathrm{~cm}$ from 20 to 60 , then every $20 \mathrm{~cm}$. Samples for molecular analyses were chosen based on TOC profiles.

\subsection{Lignin derived phenols analyses}

Lignin derived phenols were analyzed as described by Hedges and Ertel (1982). Briefly: $1 \mathrm{~g}$ of $\mathrm{CuO}, 100 \mathrm{mg}$ of $\mathrm{Fe}\left(\mathrm{NH}_{4}\right)_{2}\left(\mathrm{SO}_{4}\right)_{2} 6 \mathrm{H}_{2} \mathrm{O}, 30 \mathrm{mg}$ of plant material or $100 \mathrm{mg}$ of sediments, previously dried, were weighted. The mixture was placed in a minibomb with an agitator and a Teflon lined screw cap. Hydrolysis was carried out in $2 \mathrm{~N} \mathrm{NaOH}$ at $170^{\circ} \mathrm{C}$ for $3 \mathrm{hrs}$ under a nitrogen atmosphere. Then, the bombs were cooled at room temperature and ethylvanillin was added as internal standard $(200 \mu \mathrm{g})$. Samples were acidified at $\mathrm{pH} 1$ with $6 \mathrm{~N} \mathrm{HCl}$. After precipitation of humic acids, the samples were centrifuged at $3000 \mathrm{rpm}$ for $10 \mathrm{~min}$. Then, the lignin-derived phenols were extracted with freshly distilled ethylether. After solvent evaporation, the extracts were dried and stored in a freezer. The sample extracts were 
dissolved in pyridine, derivatised with a trimethylsilylating agent (Sylon BFT Supelco) and analyzed immediately using a Perkin Elmer gas chromatograph fitted with a $25 \mathrm{~m}$ by $0.25 \mathrm{~mm}$ i.d. DB-1 capillary column (CPSil5CB, $0.25 \mu \mathrm{m}$ film thickness) and a FID detector. The injector was maintained at $240^{\circ} \mathrm{C}$, the detector at $300^{\circ} \mathrm{C}$. Helium was the carrier gas. The analysis began at $100^{\circ} \mathrm{C}$, and then the oven temperature was raised at a rate of $4^{\circ} \mathrm{Cmin}-1$ up to $270^{\circ} \mathrm{C}$ where it was finally held for $16 \mathrm{~min}$. Compound assignment and quantification was made using internal standards and analyzing an in-lab prepared reference mix of commercial products (all analytical grade). Lignin analysis replicates gave the following precision: better than $10 \%$ for plant material and between 10 and $15 \%$ for sediments.

"Xlignin" expressed in mmol $\mathrm{C} / \mathrm{mol}$ total organic carbon, represents the total yield of 8 simple phenols: vanillic and syringic acids, aldehydes and ketones, plus two cinnamic units. This parameter does not include $p$-hydroxyl moieties that can also be derived from non vascular plant material (Goñi and Hedges, 1995). C/V ratio is defined as the molar ratio of the sum of the cinnamic phenols ( $p$-coumaric and ferulic acids) over the sum of the vanillic phenols; $S / V$ ratio is defined as the molar ratio of the sum of the syringic phenols over the sum of the vanillic phenols. (Ad/AI) is the molar ratio of vanillic acid to vanillin (aldehyde). (Ad/Al)s is the molar ratio of syringic acid to syringaldehyde.

\subsection{Neutral sugar analyses}

Neutral sugars analyses were carried out in 2 steps according to a modified Cowie and Hedges procedure (1984). The first step, which comprises a phase of soaking with $24 \mathrm{~N} \mathrm{H}_{2} \mathrm{SO}_{4}$, allows the hydrolysis of cellulose and hemicelluloses. The second step consisting of the same procedure without 24 $\mathrm{N} \mathrm{H}_{2} \mathrm{SO}_{4}$ pretreatment, only yielded hemicellulose monomers. Consequently in the data presented herein, hemicellulose content was derived from the second step whereas the cellulose content was calculated by subtraction of the results obtained in the first step from those obtained in the second step.

In a Pyrex tube, $1 \mathrm{~mL}$ of $24 \mathrm{~N} \mathrm{H}_{2} \mathrm{SO}_{4}$ was added to $30 \mathrm{mg}$ of plant material or $100 \mathrm{mg}$ of sediments, previously dried. After $12 \mathrm{hrs}$ at room temperature, samples were diluted to $1.2 \mathrm{M} \mathrm{H}_{2} \mathrm{SO}_{4}$. Tubes were tightly closed under vacuum and heated at $100^{\circ} \mathrm{C}$ for $5 \mathrm{hrs}$. After cooling, deoxy-6-glucose $(400 \mu \mathrm{g})$ was then added as an internal standard and the samples were subsequently neutralized with $\mathrm{CaCO}_{3}$. Precipitate was removed by centrifugation at 3000 rpm for $20 \mathrm{~min}$. After evaporation of the supernatant to dryness, the sugars were dissolved in $\mathrm{MeOH}$. Then, the solution was purified by centrifugation at $2500 \mathrm{rpm}$ for $5 \mathrm{~min}$ and introduced in another vessel. After evaporation of the solvent, the residue was stored frozen.

The sample extracts were dissolved in pyridine in presence of $\mathrm{LiClO}_{4}(1: 1500$ $\mathrm{w} / \mathrm{v}$ ), left $12 \mathrm{Hrs}$ at $60^{\circ} \mathrm{C}$, and then derivatized with trimethylsilyl (Sylon BFT 
Supelco) and immediately analyzed using a Perkin Elmer gas chromatograph fitted with a $25 \mathrm{~m}$ by $0.25 \mathrm{~mm}$ i.d. DB-1 capillary column (CPSil5CB, $0.25 \mu \mathrm{m}$ film thickness) and an FID detector. The temperature program began at $60^{\circ} \mathrm{C}$, then the oven temperature was raised at a rate of $30^{\circ} \mathrm{C} \mathrm{min-1}$ up to $120^{\circ} \mathrm{C}$ where it was maintained for $1 \mathrm{~min}$, then raised again to $240^{\circ} \mathrm{C}$ at $3^{\circ} \mathrm{C} \mathrm{min}-1$ and finally at a rate of $20^{\circ} \mathrm{C} \mathrm{min}-1$ up to $310^{\circ} \mathrm{C}$ were it was kept for $10 \mathrm{~min}$. The injector split was off at the start time and turned on after 2 min. The injector was maintained at $240^{\circ} \mathrm{C}$, the detector at $300^{\circ} \mathrm{C}$. Compound assignment and quantification was made using internal standards and analyzing an in-lab prepared reference mix of commercial products (all analytical grade). Replicate analyses gave an analytical precision better than $5 \%$ for neutral sugar analyses of plant material and between 10 and $15 \%$ for sediments. Eight monosaccharides were identified in vascular plant debris and in sediments: ribose, arabinose, xylose, rhamnose, fucose, glucose, mannose and galactose. Total neutral sugars are expressed in $\mathrm{mmol}$ of carbon from neutral monosaccharides per mol of total organic carbon (mmol TCOH-C/mol TOC).

\subsection{Stable carbon isotopic analyses}

${ }^{13} \mathrm{C}$ values were obtained after oxidation of the carbonate free organic matter at $850^{\circ} \mathrm{C}$ in the presence of $\mathrm{CuO}$ and $\mathrm{Cu}_{2} \mathrm{O}$ in evacuated quartz tubes. The standardisation was made against NBS 19. Measurements were performed on a VG Isogas SIRA (Serie II) spectrometer and the data plotted against $\mathrm{V}$-PDB standard. Deviations of the measured values were usually smaller than $0.15 \%$.

\section{Results}

\subsection{Plant compositions}

Wood and leaves from Avicennia geminans, Laguncularia racemosa, Rhizophora mangle, Crenea maritima, and Acrostichum aureum were analyzed for elemental, stable carbon isotope, lignin and neutral monosaccharide compositions. Results are presented in Iable 1 and in Electronic Annex EA-1. TOC values of all plant species ranged between 35 and $42 \%$. Carbon/Nitrogen ratios exceeded 50 in wood, whereas, they ranged from 18 to 28 in leaves. $\delta^{13} \mathrm{C}$ values of all higher plant species ranged between -30 and $-27 \%$, data on cyanobacteria from surface sediments of the pioneer mangrove gave a $\delta^{13} \mathrm{C}$ value close to $-19 \%$. 
Table 1. Geochemical characterization of the dominant plant species developing along the mangrove fringed coast of French Guiana.

\begin{tabular}{|c|c|c|c|c|c|c|c|c|c|}
\hline & $\begin{array}{l}\text { A. } \\
\text { gemin } \\
\text { ans leaf }\end{array}$ & $\begin{array}{l}\text { A. } \\
\text { germin } \\
\text { ans } \\
\text { wood }\end{array}$ & $\begin{array}{l}\mathbf{R} \\
\text { mang } \\
\text { le } \\
\text { leaf }\end{array}$ & $\begin{array}{l}\mathbf{R} \\
\text { mang } \\
\text { le } \\
\text { wood }\end{array}$ & $\begin{array}{l}\text { L } \\
\text { racem } \\
\text { osa } \\
\text { leaf }\end{array}$ & $\begin{array}{l}\text { L } \\
\text { racem } \\
\text { osa } \\
\text { wood }\end{array}$ & $\begin{array}{l}\text { C. } \\
\text { maniti } \\
\text { ma } \\
\text { leaf }\end{array}$ & $\begin{array}{l}\text { C. } \\
\text { mariti } \\
\text { ma } \\
\text { wood }\end{array}$ & $\begin{array}{l}\text { A. } \\
\text { aureu } \\
\text { m }\end{array}$ \\
\hline TOC (\%) & 40.7 & 42.7 & 40.3 & 40.5 & 41.9 & 40.6 & 35.5 & 38.8 & 42.0 \\
\hline $\mathrm{C} / \mathrm{N}$ & 18.2 & 55.5 & 29.0 & 57.2 & 19.3 & 53.2 & 22.2 & 58.5 & 25.3 \\
\hline$\delta^{13} \mathrm{C}(\% \circ)$ & -27.9 & -28 & -30.5 & -30.1 & -29.5 & -30.4 & -28.6 & -27.6 & -26.8 \\
\hline $\begin{array}{l}\text { X lignine } \\
\text { (mmol } \\
\text { phenolic C } \\
\text { / mol TOC) }\end{array}$ & 14.6 & 38.8 & 11.3 & 45.4 & 10.5 & 50.4 & 5.2 & 51.2 & 31.2 \\
\hline $\begin{array}{l}\sum 11 \text { (mmol } \\
\text { phenolic C } \\
\text { / mol TOC) }\end{array}$ & 20.8 & 42.0 & 22.6 & 51.2 & 20.0 & 53.88 & 13.4 & 54.4 & 48.9 \\
\hline C/V & 1.58 & 0.04 & 4.14 & 0.02 & 2.00 & 0.02 & 0.15 & 0.0 & 0.06 \\
\hline$S / V$ & 1.04 & 1.78 & 1.50 & 2.38 & 1.76 & 2.28 & 0.61 & 0.72 & 0.42 \\
\hline$(\mathrm{Ad} / \mathrm{Al}) \vee$ & 0.45 & 0.03 & 0.47 & 0.02 & 0.45 & 0.03 & 0.35 & 0.02 & 0.02 \\
\hline$(\mathrm{Ad} / \mathrm{Al}) \mathrm{s}$ & 0.79 & 0.32 & 0.18 & 0.21 & 0.64 & 0.17 & 0.29 & 0.21 & 0.0 \\
\hline $\begin{array}{l}\text { Total } \\
\text { Carbohydr } \\
\text { ates (mmol } \\
\text { TCHO-C / } \\
\text { mol TOC) }\end{array}$ & 155.2 & 253.7 & $\begin{array}{l}127.5 \\
9\end{array}$ & 364.2 & 145.0 & 354.9 & 319.4 & 353.9 & 230.1 \\
\hline $\begin{array}{l}\text { Cellulosic } \\
\text { Glucose } \\
(\%)\end{array}$ & 45 & 41 & 42 & 50 & 44 & 52 & 28 & 50 & 29 \\
\hline $\begin{array}{l}\text { Hemicellulo } \\
\text { sic Glucose } \\
(\%)\end{array}$ & 11 & 6 & 13 & 3 & 11 & 9 & 48 & 12 & 36 \\
\hline $\begin{array}{l}\text { Total } \\
\text { Ribose (\%) }\end{array}$ & $<1$ & $<1$ & $<1$ & $<1$ & $<1$ & $<1$ & $<1$ & $<1$ & $<1$ \\
\hline $\begin{array}{l}\text { Total } \\
\text { Arabinose } \\
\text { (\%) }\end{array}$ & 6 & 13 & 14 & 9 & 16 & 6 & 3 & 3 & 3 \\
\hline $\begin{array}{l}\text { Total Xylose } \\
\text { (\%) }\end{array}$ & 18 & 24 & 6 & 23 & 6 & 23 & 4 & 23 & 11 \\
\hline $\begin{array}{l}\text { Total } \\
\text { Rhamnose } \\
\text { (\%) }\end{array}$ & 9 & 7 & 6 & 2 & 5 & 2 & 4 & 2 & 3 \\
\hline
\end{tabular}




\begin{tabular}{|l|l|l|l|l|l|l|l|l|l|}
\hline & $\begin{array}{l}\text { A. } \\
\text { gemin } \\
\text { ans leaf }\end{array}$ & $\begin{array}{l}\text { A. } \\
\text { gemin } \\
\text { ans } \\
\text { wood }\end{array}$ & $\begin{array}{l}\text { R } \\
\text { mang } \\
\text { le } \\
\text { leaf }\end{array}$ & $\begin{array}{l}\text { R. } \\
\text { mang } \\
\text { le } \\
\text { wood }\end{array}$ & $\begin{array}{l}\text { L } \\
\text { racem } \\
\text { osa } \\
\text { leaf }\end{array}$ & $\begin{array}{l}\text { L } \\
\text { racem } \\
\text { osa } \\
\text { wood }\end{array}$ & $\begin{array}{l}\text { C. } \\
\text { maniti } \\
\text { ma } \\
\text { leaf }\end{array}$ & $\begin{array}{l}\text { C. } \\
\text { maniti } \\
\text { ma } \\
\text { wood }\end{array}$ & $\begin{array}{l}\text { A. } \\
\text { aureu } \\
\text { m }\end{array}$ \\
\hline $\begin{array}{l}\text { Total } \\
\text { Fucose (\%) }\end{array}$ & 1 & $<1$ & 5 & 4 & 2 & $<1$ & 1 & 1 & 1 \\
\hline $\begin{array}{l}\text { Total } \\
\text { Mannose } \\
\text { (\%) }\end{array}$ & 5 & 4 & 4 & 3 & 6 & 4 & 4 & 5 & 10 \\
\hline $\begin{array}{l}\text { Total } \\
\text { Galactose } \\
\text { (\%) }\end{array}$ & 4 & 5 & 9 & 5 & 9 & 4 & 8 & 4 & 7 \\
\hline $\begin{array}{l}\text { Hemicellulo } \\
\text { sic } \\
\text { carbohydr } \\
\text { ates (\%) }\end{array}$ & 48 & 50 & 55 & 37 & 41 & 36 & 69 & 41 & 43 \\
\hline
\end{tabular}

Percent of total organic carbon (TOC), carbon/nitrogen ratios $(C / N)$, stable carbon isotope $\left(\delta^{13} \mathrm{C}\right.$ in \%o PDB), Xlignin (sum of the vanillic, syringic and cinnamic phenols in mmol phenolic $\mathrm{C} / \mathrm{mol}$ total OC), $\Sigma 11$ (sum of $X_{\text {lignin }}$ and p-hydroxyl phenols in $\mathrm{mmol}$ phenolic $\mathrm{C} / \mathrm{mol}$ total $\mathrm{OC}$ ), cinnamic/vanillic ratios (C/V), syringic/vanillic ratios $(S / V)$, vanillic acid/vanillicratios $(\mathrm{Ad} / \mathrm{Al})_{\mathrm{v}}$, syringic acid/syringaldehyde (Ad/Al)s; total neutral sugars in $\mathrm{mmol} \mathrm{TCHO}-\mathrm{C} / \mathrm{mol}$ total OC; cellulose glucose, hemicellulosic glucose, total ribose, total arabinose, total xylose, total rhamnose, total fucose, total mannose and total galactose in \% of total carbohydrates; hemicellulosic carbohydrates in \% of total carbohydrates.

TOC normalized yields of phenols released by CuO oxidation showed great variations between leaves and wood of plant species composing the mangroves of French Guiana. Woods were richer than leaves in lignin oxidation product (LOP). Xlignin (see experimental) ranged from 5 to 15 and from 38 to $51 \mathrm{mmol} \mathrm{C} / \mathrm{mol}$ total organic carbon in leaves and wood material, respectively. C/V ratio is close to 0 for wood of every mangrove. C/V ratios ranged between 1.5 and 4 for leaves of Rhizophora mangle, Avicennia geminans and Lagunc ularia racemosa. For all species, S/V ratios were higher for woody tissues, ranging from 0.7 to 2.3, than for herbaceous tissues, ranging from 0.6 to 1.7. (Ad/Al)v and (Ad/Al)s were higher in leaves, from 0.3 to 0.4 and from 0.3 to 0.7 respectively, than in woody tissues, where they are close to 0 and from 0.2 to 0.3 respectively, except for $\mathrm{R}$. mangle, for which both ratios were close to 0.2 .

Woody tissues were richer than leaves in neutral sugars, i.e., 250 to 360 and 120 to $320 \mathrm{mmol} \mathrm{TCOH}-\mathrm{C} / \mathrm{mol} \mathrm{TOC}$, respectively. Glucose was the most abundant neutral sugar in every plant species, representing more than $50 \%$ of the total; $80 \%$ of total glucose was cellulosic glucose. Except for glucose and mannose, the other neutral sugars were mostly -and in some cases exclusively -hemicellulosic. In woods, cellulosic glucose and xylose represented individually more than $20 \%$ of total sugars, whereas the proportions of other neutral sugars varied as follows: arabinose (3 to 16\%), galactose (4 to 9\%), 
rhamnose ( 2 to $9 \%$ ), mannose (2 to $10 \%$ ), fucose $(0.2$ to $5 \%$ ) and ribose $(<1 \%)$. The composition of the fern $A$. a ureum differs from that of the other plants, with lower content in cellulosic glucose and in xylose.

\subsection{Sedimentary cores from the various mangroves}

Results concerning sediments collected beneath the various mangrove stands are presented in Figure 2, Table 2 and Electronic Annex AE-2. for neutral carbohydrates, and Table 3 and Electronic Annex AE-3. for lignin derived phenols. Total organic carbon varied between 0.7 and 13\%, with maximum values increasing from the young mature to the senescent mangrove. TOC values were maximum in the upper $5 \mathrm{~cm}$ and decreased with depth except in the "recolonized cemetery" where it increased at 30 and $65 \mathrm{~cm}$ deep. These depths were also characterized by an increase in $\mathrm{C} / \mathrm{N}$ ratios, which varied from 8 to 25 with maximum values between 5 and 20 $\mathrm{cm}$ depth. $\delta^{13} \mathrm{C}$ values showed an inverse pattern, ranging from -29 to -23 $\%$. Total carbohydrates ranged between 20 and $90 \mathrm{mmol}$ TCHO-C / mol TOC, and $X_{\text {lignin }}$ ranged between 20 and $80 \mathrm{mmol}$ phenolic C / mol TOC. In most cores, Xlignin total neutral monosaccharides, total xylose, and total cellulosic monosaccharides reached a maximum at 5 to $20 \mathrm{~cm}$ depth of most cores.

In every stages of mangrove development, hemicellulosic glucose content was high on the whole depth profile, whereas cellulosic glucose content was maximum in the upper core and decreased with depth except in the senescent mangrove where it is elevated whatever the depth. Concerning the Avicennia and Rhizophora young mature mangrove and the dead recolonized mangrove, which are all flooded at each tide, rhamnose content was elevated at sediment surface, with values close to $20 \%$ of total carbohydrates. In cores collected in the young mature, mature, and senescent Avicennia dominated mangrove, total carbohydrates increased beneath $40 \mathrm{~cm}$ deep, mainly reflecting an increase in fucose and rhamnose content, up to 27 and $43 \%$ respectively. In the upper core, these deoxy carbohydrates, as well as arabinose, were stable and in high content compared to their concentration in mangrove plants. Total mannose and total galactose content were low, $<10 \%$, and stable on the whole depth profile of every cores. 


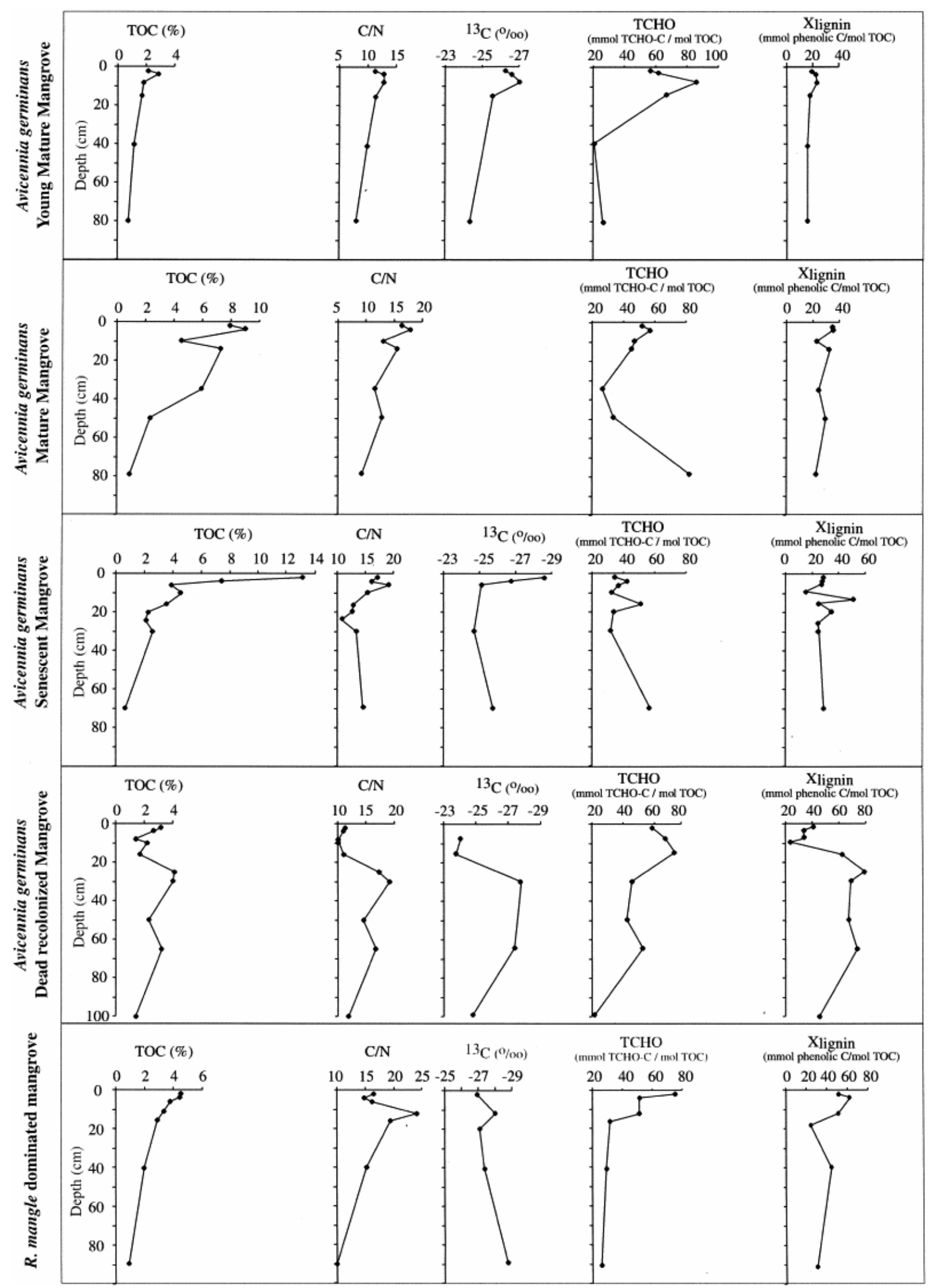

Fig. 2. Depth profiles from the different stages of the A. geminans mangrove swamps, i.e., young mature mangrove, mature mangrove, senescent mangrove, recolonized cemetery mangrove, and from the R. mangle young mature mangrove: percent total organic carbon (TOC), carbon/nitrogen ratios $(\mathrm{C} / \mathrm{N})$, stable carbon isotope $\left(\delta^{13} \mathrm{C}\right.$ in \%० $\left.\mathrm{PDB}\right)$, sum of the eight monosaccharides (TCHO) (ribose, arabinose, xylose, rhamnose, fucose glucose mannose and galactose) in $\mathrm{mmol} \mathrm{TCHO}-\mathrm{C} / \mathrm{mol}$ total $\mathrm{OC}$ and $\mathrm{X}$ lignin (sum of the vanillic syringic and cinnamic phenols in $\mathrm{mmol}$ phenolic $\mathrm{C} / \mathrm{mol}$ total $\mathrm{OC}$ ). 
Table 2. : Neutral carbohydrates depth distribution in the different stages of the A. germinans mangrove swamps, i.e. young mature mangrove, senescent mangrove, recolonized cemetary mangrove, and in the $R$. mangle young mature mangrove.

\begin{tabular}{|c|c|c|c|c|c|c|c|c|c|c|c|c|}
\hline $\begin{array}{l}\text { Mang } \\
\text { rove }\end{array}$ & $\begin{array}{l}\text { De } \\
\text { pt } \\
\text { h } \\
\text { (c } \\
\text { m) }\end{array}$ & $\begin{array}{l}\text { Total } \\
\text { Carboh } \\
\text { ydrates } \\
\text { (mmol } \\
\text { TCHO- } \\
\text { C / mol } \\
\text { TOC) }\end{array}$ & $\begin{array}{l}\text { Cell } \\
\text { ulosi } \\
\text { C } \\
\text { Gluc } \\
\text { ose } \\
\text { (\%) }\end{array}$ & $\begin{array}{l}\text { Hemic } \\
\text { ellulosi } \\
\text { c } \\
\text { Glucos } \\
\text { e (\%) }\end{array}$ & $\begin{array}{l}\text { Tot } \\
\text { al } \\
\text { Rib } \\
\text { os } \\
\text { e } \\
\text { (\%) }\end{array}$ & $\begin{array}{l}\text { Total } \\
\text { Arab } \\
\text { inos } \\
\text { e (\%) }\end{array}$ & $\begin{array}{l}\text { Tot } \\
\text { al } \\
\text { Xyl } \\
\text { os } \\
\text { e } \\
\text { (\%) }\end{array}$ & $\begin{array}{l}\text { Total } \\
\text { Rha } \\
\text { mnos } \\
\text { e (\%) }\end{array}$ & $\begin{array}{l}\text { Tot } \\
\text { al } \\
\text { Fuc } \\
\text { ose } \\
\text { (\%) }\end{array}$ & $\begin{array}{l}\text { Total } \\
\text { Man } \\
\text { nos } \\
\text { e } \\
\text { (\%) }\end{array}$ & $\begin{array}{l}\text { Total } \\
\text { Gala } \\
\text { ctos } \\
\text { e (\%) }\end{array}$ & $\begin{array}{l}\text { Hemic } \\
\text { ellulosi } \\
\text { c } \\
\text { carboh } \\
\text { ydrates } \\
(\%)\end{array}$ \\
\hline & 2 & 56.7 & 18 & 17 & $<1$ & 12 & 11 & 29 & 3 & 5 & 5 & 80 \\
\hline $\begin{array}{l}\text { A. } \\
\text { germi } \\
\text { nans }\end{array}$ & 4 & 63.1 & 20 & 17 & $<1$ & 8 & 14 & 27 & 3 & 4 & 6 & 66 \\
\hline $\begin{array}{l}\text { Youn } \\
\mathrm{g}\end{array}$ & 8 & 86.8 & 11 & 18 & $<1$ & 16 & 17 & 25 & 3 & 4 & 5 & 61 \\
\hline $\begin{array}{l}\text { Matur } \\
\mathrm{e}\end{array}$ & 15 & 67.0 & 0 & 24 & $<1$ & 21 & 12 & 25 & 3 & 5 & 7 & 99 \\
\hline \multirow[t]{4}{*}{$\begin{array}{l}\text { Mang } \\
\text { rove }\end{array}$} & 40 & 20.5 & 0 & 16 & - & 21 & 42 & 13 & - & 4 & 4 & 92 \\
\hline & 80 & 25.9 & 0 & 15 & $<1$ & 16 & 14 & 18 & 27 & 2 & 7 & 92 \\
\hline & 2 & 52.2 & 36 & 12 & $<1$ & 14 & 10 & 9 & 7 & 7 & 6 & 36 \\
\hline & 4 & 57.4 & 25 & 18 & 1 & 17 & 15 & 9 & 6 & 5 & 6 & 76 \\
\hline $\begin{array}{l}\text { A. } \\
\text { germi } \\
\text { nans }\end{array}$ & 10 & 47.2 & 12 & 27 & 1 & 16 & 14 & 11 & 7 & 6 & 7 & 87 \\
\hline $\begin{array}{l}\text { Matur } \\
\mathrm{e}\end{array}$ & 14 & 45.1 & 34 & 10 & 1 & 16 & 10 & 10 & 6 & 8 & 4 & 39 \\
\hline \multirow[t]{6}{*}{$\begin{array}{l}\text { Mang } \\
\text { rove }\end{array}$} & 35 & 26.4 & 18 & 15 & 2 & 18 & 13 & 21 & 8 & 3 & 3 & 80 \\
\hline & 50 & 33.7 & 7 & 15 & 1 & 18 & 8 & 29 & 13 & 4 & 5 & 91 \\
\hline & 80 & 82.3 & 7 & 4 & 2 & 19 & 6 & 43 & 13 & 4 & 3 & 92 \\
\hline & 2 & 34.4 & 21 & 24 & $<1$ & 18 & 9 & 13 & 4 & 5 & 5 & 64 \\
\hline & 4 & 43.1 & 33 & 12 & $<1$ & 15 & 9 & 14 & 3 & 6 & 7 & 61 \\
\hline & 6 & 37.4 & 28 & 11 & 1 & 17 & 11 & 19 & 5 & 5 & 4 & 51 \\
\hline $\begin{array}{l}\text { A. } \\
\text { germi } \\
\text { nans }\end{array}$ & 10 & 32.4 & 22 & 14 & $<1$ & 21 & 10 & 19 & 5 & 4 & 5 & 77 \\
\hline
\end{tabular}




\begin{tabular}{|c|c|c|c|c|c|c|c|c|c|c|c|c|}
\hline $\begin{array}{l}\text { Mang } \\
\text { rove }\end{array}$ & $\begin{array}{l}\text { De } \\
\text { pt } \\
\text { h } \\
\text { (c } \\
\text { m) }\end{array}$ & $\begin{array}{l}\text { Total } \\
\text { Carboh } \\
\text { ydrates } \\
\text { (mmol } \\
\text { TCHO- } \\
\text { C / mol } \\
\text { TOC) }\end{array}$ & $\begin{array}{l}\text { Cell } \\
\text { ulosi } \\
\text { c } \\
\text { Gluc } \\
\text { ose } \\
(\%)\end{array}$ & $\begin{array}{l}\text { Hemic } \\
\text { ellulosi } \\
\text { c } \\
\text { Glucos } \\
\text { e (\%) }\end{array}$ & $\begin{array}{l}\text { Tot } \\
\text { al } \\
\text { Rib } \\
\text { os } \\
\text { e } \\
\text { (\%) }\end{array}$ & $\begin{array}{l}\text { Total } \\
\text { Arab } \\
\text { inos } \\
\text { e (\%) }\end{array}$ & $\begin{array}{l}\text { Tot } \\
\text { al } \\
\text { Xyl } \\
\text { os } \\
\text { e } \\
\text { (\%) }\end{array}$ & $\begin{array}{l}\text { Total } \\
\text { Rha } \\
\text { mnos } \\
\text { e (\%) }\end{array}$ & $\begin{array}{l}\text { Tot } \\
\text { al } \\
\text { Fuc } \\
\text { ose } \\
\text { (\%) }\end{array}$ & $\begin{array}{l}\text { Total } \\
\text { Man } \\
\text { nos } \\
\text { e } \\
\text { (\%) }\end{array}$ & $\begin{array}{l}\text { Total } \\
\text { Gala } \\
\text { ctos } \\
\text { e (\%) }\end{array}$ & $\begin{array}{l}\text { Hemic } \\
\text { ellulosi } \\
\text { c } \\
\text { carboh } \\
\text { ydrates } \\
(\%)\end{array}$ \\
\hline $\begin{array}{l}\text { Senes } \\
\text { cent }\end{array}$ & 16 & 51.7 & 47 & 13 & $<1$ & 13 & 10 & 8 & 2 & 2 & 4 & 36 \\
\hline \multirow[t]{4}{*}{$\begin{array}{l}\text { Mang } \\
\text { rove }\end{array}$} & 20 & 34.0 & 11 & 21 & $<1$ & 22 & 14 & 16 & 6 & 3 & 6 & 78 \\
\hline & 30 & 32.1 & 30 & 15 & $<1$ & 16 & 8 & 20 & 5 & 2 & 4 & 70 \\
\hline & 70 & 56.9 & 25 & 10 & - & 15 & 8 & 14 & 14 & 8 & 5 & 42 \\
\hline & 2 & 60.5 & 10 & 11 & $<1$ & 15 & 28 & 25 & 2 & 4 & 4 & 87 \\
\hline $\begin{array}{l}\text { A. } \\
\text { germi } \\
\text { nans }\end{array}$ & 8 & 69.5 & 11 & 19 & 4 & 20 & 11 & 16 & 2 & 7 & 10 & 73 \\
\hline Dead & 15 & 75.8 & 15 & 25 & 1 & 22 & 12 & 9 & 7 & 5 & 5 & 80 \\
\hline $\begin{array}{l}\text { Recol } \\
\text { onize } \\
d\end{array}$ & 30 & 46.8 & 36 & 12 & $<1$ & 15 & 12 & 12 & 4 & 4 & 5 & 40 \\
\hline \multirow[t]{4}{*}{$\begin{array}{l}\text { Mang } \\
\text { rove }\end{array}$} & 50 & 43.7 & 37 & 5 & - & 21 & 10 & 12 & 4 & 3 & 7 & 30 \\
\hline & 65 & 54.1 & 19 & 17 & $<1$ & 23 & 10 & 13 & 6 & 6 & 5 & 47 \\
\hline & $\begin{array}{l}10 \\
0\end{array}$ & 21.9 & 4 & 38 & - & 18 & 9 & 19 & 4 & 3 & 3 & 78 \\
\hline & 2 & 75.9 & 23 & 6 & $\begin{array}{l}20 \\
0\end{array}$ & 23 & 11 & 19 & 5 & 5 & 7 & 65 \\
\hline $\begin{array}{l}\text { R. } \\
\text { mang } \\
\text { le }\end{array}$ & 4 & 51.3 & 37 & 13 & $<1$ & 15 & 11 & 10 & 5 & 3 & 6 & 51 \\
\hline $\begin{array}{l}\text { Youn } \\
\mathrm{g}\end{array}$ & 12 & 51.5 & 23 & 7 & $\begin{array}{l}10 \\
0\end{array}$ & 24 & 12 & 14 & 6 & 6 & 8 & 29 \\
\hline $\begin{array}{l}\text { Matur } \\
\mathrm{e}\end{array}$ & 18 & 31.7 & 14 & 19 & $<1$ & 22 & 8 & 18 & 8 & 7 & 3 & 62 \\
\hline \multirow[t]{2}{*}{$\begin{array}{l}\text { Mang } \\
\text { rove }\end{array}$} & 40 & 29.5 & 8 & 16 & - & 23 & 10 & 19 & 11 & 7 & 5 & 74 \\
\hline & 90 & 25.9 & 0 & 18 & - & 20 & 11 & 21 & 10 & 17 & 4 & 60 \\
\hline
\end{tabular}

Total neutral sugars in mmol TCHO-C/mol total OC; cellulosic glucose, hemicellulosic glucose, total ribose, total arabinose, total xylose, total rhamnose, total fucose, total mannose and 
total galactose in \% of total carbohydrates; hemicelluosic carbohydrates in \% of total carbohydrates.

Table 3. : Some specific monomeric lignin ratios depth distribution in the different stages of the A. germinans mangrove swamps, i.e. young mature mangrove, mature mangrove, senescent mangrove, recolonized cemetery mangrove, and in the $R$. mangle young mature mangrove.

\begin{tabular}{|c|c|c|c|c|c|}
\hline & Depth $(\mathrm{cm})$ & $\mathrm{C} / \mathrm{V}$ & S/V & $(\mathrm{Ad} / \mathrm{Al}) \mathrm{v}$ & (Ad/Al)s \\
\hline & 2 & 0.13 & 1.16 & 0.38 & 1.37 \\
\hline A geminans & 4 & 0.08 & 1.18 & 0.23 & 0.21 \\
\hline Young & 8 & 0.04 & 1.45 & 0.35 & 0.43 \\
\hline Mature & 15 & 0.37 & 0.69 & 1.32 & 0.50 \\
\hline \multirow[t]{4}{*}{ Mangrove } & 40 & 0.36 & 0.26 & - & - \\
\hline & 80 & 0.53 & 0.00 & - & - \\
\hline & 2 & 0.31 & 2.06 & 0.10 & 0.71 \\
\hline & 4 & 0.19 & 1.85 & 0.06 & 0.45 \\
\hline A. geminans & 10 & 0.24 & 0.86 & 0.10 & 1.68 \\
\hline Mature & 14 & 0.06 & 1.19 & 0.04 & 0.45 \\
\hline \multirow[t]{5}{*}{ Mangrove } & 35 & 0.21 & 1.28 & 0.09 & 0.45 \\
\hline & 50 & - & 1.39 & 0.46 & 0.44 \\
\hline & 80 & - & 0.99 & 1.24 & - \\
\hline & 2 & 0.25 & 1.20 & 0.07 & 0.64 \\
\hline & 4 & 0.19 & 1.13 & 0.05 & 0.49 \\
\hline A. germinans & 6 & 0.07 & 0.97 & 0.11 & 1.14 \\
\hline Senescent & 10 & 0.04 & 0.67 & 0.09 & 1.44 \\
\hline \multirow[t]{7}{*}{ Mangrove } & 14 & 0.27 & 1.13 & 0.95 & 0.69 \\
\hline & 16 & - & 1.25 & 0.14 & 2.21 \\
\hline & 20 & 0.27 & 1.21 & 0.13 & 1.56 \\
\hline & 25 & - & 1.01 & 0.17 & 1.67 \\
\hline & 30 & - & 0.83 & 0.20 & 2.34 \\
\hline & 70 & 0.07 & 1.10 & 0.78 & 1.57 \\
\hline & 2 & 0.06 & 1.20 & 0.15 & 0.41 \\
\hline
\end{tabular}




\begin{tabular}{|l|l|l|l|l|l|}
\hline & Depth $(\mathbf{c m})$ & $\mathbf{C} / \mathbf{V}$ & $\mathbf{S} / \mathbf{V}$ & $\mathbf{( A d} / \mathbf{A l}) \mathbf{v}$ & (Ad/Al)s \\
\hline & 4 & 0.34 & 0.96 & 0.38 & 0.93 \\
\hline A. geminans & 8 & 0.15 & 1.55 & 0.71 & 0.43 \\
\hline Dead & 10 & - & 1.45 & 0.41 & 0.15 \\
\hline Recolonized & 15 & 0.10 & 2.02 & 0.29 & 0.71 \\
\hline Mangrove & 25 & 0.02 & 0.90 & 0.01 & 1.07 \\
\hline & 30 & 0.01 & 1.87 & 0.08 & 0.50 \\
\hline & 50 & 0.03 & 2.00 & 0.15 & 0.31 \\
\hline & 65 & 0.02 & 1.44 & 0.38 & 0.32 \\
\hline & 100 & - & 1.62 & 0.31 & 0.35 \\
\hline & 2 & $<0.01$ & 1.79 & 0.06 & 0.32 \\
\hline R. mangle & 4 & - & 1.83 & 0.05 & 0.39 \\
\hline Young & 12 & - & 1.76 & 0.05 & 0.46 \\
\hline Mature & 18 & - & 1.25 & 0.18 & 0.30 \\
\hline Mangrove & 40 & - & 1.92 & 0.22 & 0.52 \\
\hline & 90 & - & 1.43 & 0.51 & 0.46 \\
\hline
\end{tabular}

Cinnamic/vanillic ratios $(C / V)$, syringic/vanillic ratios $(S / V)$, vanillic acid/vanillin ratios $(A d / A I)_{v}$, syringic acid/syringaldehyde (Ad/Al)s.

$\mathrm{C} / \mathrm{V}$ were very low, $<0.6$ with mean values close to 0.1 , in every stages of mangrove development, and reached a minimum at depths characterized by a maximum in $X_{\text {lignin, }}$ total neutral monosaccharides, total xylose, and total cellulosic monosaccharides. S/V ratio was low with values $<2$ in every stages of mangrove development. (Ad/Al) ratios of both syringic and vanillic units were high without any obvious pattern in the upper core collected in the younger Avicennia mangroves whereas in the older stages and in the Rhizophora mangrove, only the (Ad/Al) of the syringic unit was elevated, (Ad/Al) of the vanillic units was lower than 0.1. At depth, the (Ad/Al) of the vanillic unit increased in all cores. 


\section{Disc ussion}

\subsection{Comparative plant composition}

No consistent compositional differences among angiosperm trees were evident. In contrast, clear distinction can be made between leaves and woods. $\delta^{13} \mathrm{C}$ values, ranging from -30 and $-27 \%$, were close to those previously reported in other mangroves (Lallier-Vergès et al., 1998; Dittmar and Lara, 2001; Bouillon et al., 2003) and in plants of the Amazon region (Hedges et al., 1986). ${ }^{13} \mathrm{C}$ values of cyanobacteria $(-19 \%)$ were also consistent with observations reported in the literature (Meyers, 1994). Mean $\mathrm{C} / \mathrm{N}$ ratios were in the global range for mangrove plant species, with values close to 20 for leaves and close to 50 for wood.

Few studies deal with the lignin composition of mangrove plant species (Hedges and Parker, 1976; Benner et al., 1990; Opsahl and Benner, 1995; Lallier-Vergès et al. 1999; Dittmar and Lara, 2001). Woods of Avicennia geminans, Laguncularia racemosa, Rhizophora mangle and Crenea maritima exhibited a typical vascular-plant lignin signature (Hedges and Mann, 1979a). Nonetheless, R. mangle leaves were characterized by a higher $\mathrm{C} / \mathrm{V}$ ratio, up to 4 , than other dicotyledonous angiosperm leaves, reflecting their low content in vanillic units as observed by Dittmar and Lara (2001). Fresh leaves of A. geminans presented a lignin yield of $14 \%$, consistent with observations made by Dittmar and Lara (2001). However, the leaves of Laguncularia racemosa and Rhizophora mangle indicated values close to $11 \%$, higher than those reported by Dittmar and Lara (2001) but similar to those reported by Benner et al. (1990). The acid to aldehyde ratios of both vanillic and syringic phenols which may be useful diagenetic indicators, were in the same range or higher than those reported by Dittmar and Lara (2001) and by Opsahl and Benner (1995). However, intraspecific genetic variations, resulting in different chemical compositions, can be important in mangrove plant species (Dodd et al., 1998).

Neutral monosaccharide contents varies widely among vascular plants (Aspinall, 1970), and some general trends may be observed among dicotyledonous angiosperms. Moers et al. (1990) reviewed the composition of cell walls of dicotyledonous angiosperms: primary cell walls were essentially composed of pectic and hemicellulosic polysaccharides (rhamnogalacturonans, arabinans, galactans and arabinogalactans) whereas the secondary cell walls (xylem) consisted of $40 \%-60 \%$ cellulose.

Whatever the species, our results showed great variations between neutral carbohydrates composition of wood and leaves. R. mangle wood was mainly composed of cellulosic glucose (50\%), xylose (23\%) and arabinose $(9 \%)$, consistent with Moers et al. (1990), but hemicellulosic glucose and galactose represented only 3 and $5 \%$, respectively. Whereas in R. mangle leaves hemicellulosic glucose represented more than 10\%, cellulosic glucose 
represented $42 \%$, xylose only $6 \%$ and arabinose up to $14 \%$. Our compositional pattern in R. mangle leaves was similar to those determined by Benner et al. (1990): glucose>arabinose>galactose>rhamnose>xylose. Concerning A. geminans, yields and compositional pattern of leaves and wood were, for the most part, similar to those reported by Opsahl and Benner (1999), except a slight difference in arabinose and xylose content. The main point was that Avic ennia leaves were richer in xylose (18\%) compared to other leaves from mangrove plant species.

To summarize, woods of mangrove plants were characterized by high $\mathrm{C} / \mathrm{N}$ ratios, high $\mathrm{X}_{\text {lignin }} \mathrm{C} / \mathrm{V}$ ratios close to 0 , low $(\mathrm{Ad} / \mathrm{Al})_{v}$, high content in xylose and in cellulosic glucose and low content in galactose and hemicellulosic glucose.

\subsection{Sedimentary organic matter}

The following discussion differentiates the upper $30 \mathrm{~cm}$ layer, which is mainly composed of autochthonous OM, and the basal deposits, mainly composed of allochthonous oxidized debris that originates from Amazon watershed.

\subsubsection{Autochthonous carbohydrate distributions as witnesses of OM sources}

Although carbohydrates may be protected from decomposition in sediments either by complexation with metal ions (Zn, Cu, Fe, Al) and/or by adsorption to clays (Stevenson, 1994; Bergamashi et al., 1997), in studied sediments, they were rapidly degraded at the sediment water interface in the young mangroves, or in surface litter in the older ones. Total carbon from neutral carbohydrates represented between 15 and $35 \%$ of total organic carbon (TOC) in mangrove plant species and less than $9 \%$ in mangrove sediments whatever the mangrove forest stage or environmental conditions, thus highlighting the reactivity of these components relative to bulk OC. This result is consistent with previous studies that observed little in situ degradation below the surface mixed layer (Cowie and Hedges, 1984; Stout et al. 1988). In a Rhizophora peat swamp in Florida, degradation of polysaccharides by microorganisms appeared to be restricted to the initial stages of peat formation (Moers et al., 1990). Despite a rather low content, neutral carbohydrate composition from sediment cores showed selective degradation and gave specific details on OM sources.

Even though vascular plant tissues have typical carbohydrate signatures (Cowie and Hedges, 1984), in natural media these signatures tend to disappear rapidly since degraders such as bacteria and fungi, in turn synthesize new polysaccharides. Consequently, different interpretations can be derived from carbohydrate composition. Therefore, the sources were assessed by comparing the compositions of sedimentary carbohydrates with $\mathrm{C} / \mathrm{N}$ ratios and $\delta^{13} \mathrm{C}$ values. 
In the upper part of all cores, between 5 and $20 \mathrm{~cm}$ depth, $\mathrm{C} / \mathrm{N}$ ratios increased, sometimes correlated with a decrease of $\delta^{13} \mathrm{C}$ values (Fig. 2). This result suggests the introduction of fresh OM from vascular plants. Formally, this could be OM derived from roots or from litter buried by crabs. Neutral sugar composition helps decipher between these two possibilities. The high xylose and cellulosic glucose content in the considered sedimentary level support an inference of root development rather than buried litter as primary source of new OM. Actually, A. geminans has a specific root system developing radially close to the sediment surface. In sediments, glucose was mainly hemicellulosic, whereas in mangrove plants, glucose was mainly cellulosic. Inasmuch as cellulose is more refractory to degradation than hemicellulose that can be rapidly removed by microbial hydrolysis (Stout et al., 1988), selective preservation of hemicellulosic glucose in the sediment can be ruled out. In situ synthesis of hemicellulosic type glucose in sediment by microorganisms combined with an intense decomposition of cellulose derived from higher plants may explain this trend. This result suggests that cellulose, which is highly resistant to enzymatic hydrolysis (Béguin and Aubert, 1994), could be rapidly degraded even in aquatic environment, as observed by Benner et al 1984a and Benner et al 1984b and Benner and Hodson (1985). The higher content in hemicellulosic glucose in sediments beneath the older mangrove swamps may probably result from a higher input due to litter accumulation. In young mangrove swamps, neutral carbohydrate composition depth profiles differ from those of the older swamps by a higher content in rhamnose at the top of the core. These swamps are flooded at each tide, litter is exported (Marchand et al., 2003) and diatoms, cyanophyceae and micro-green algae (De Wit, oral communication) develop at the sediment surface. Rhamnose enrichment is probably due to the algal mats that are predominantly composed of polysaccharides rich in deoxy sugars (Nierop et al., 2001). Ogier et al. (2001) observed high rhamnose (up to 50\%) contents in trap material and sediments from a lake primarily rich in diatoms and cyanophyceae. In most mangrove swamps, the upper sediment was rich in arabinose, rhamnose and fucose, compared to mangrove plants, suggesting that these sugars are produced in sediments by fungi or microbes. Previous work indicated that a high concentration of deoxy sugars could effectively be derived from bacteria (Hedges et al., 1988) but possibly also from plankton preferentially preserved in sediments (Hicks et al., 1994). Plankton and bacteria which both can contribute significantly to mangrove peats (Moers et al., 1990) could not be differentiated by their carbohydrate composition (Cowie and Hedges, 1984). Whatever mangrove forest stage or sediment depth, mannose and galactose, which both represent $\sim 5 \%$ OC, were lost at the same rate as bulk $\mathrm{OM}$, thus suggesting that they are strictly inherited from higher plants. 


\subsubsection{Autochthonous lignin distributions as witnesses of environmental conditions}

Lignin oxidation products were lost at a lower rate than total neutral sugars and bulk OC. Total carbon from lignin derived phenols represented less than $5 \%$ TOC in mangrove plant and varied between 2 and $17 \%$ in sediments, thus highlighting the lignin relative stability as previously observed (Benner et al 1984a and Benner et al 1984b; Hedges et al., 1985; Cowie et al., 1992; LallierVergès et al., 1999). Nevertheless, decomposition of lignin occurred and phenols distribution varied between sites and with depth. The next paragraph aims to identify changes in this distribution in relation to redox conditions, and thus to stages in mangrove development. Concerning the younger development stage of Avic ennia geminans (C1), C/V ratios are low, close to 0.1 , confirming the introduction of $\mathrm{OM}$ by the cable root system. S/V were stable in the upper core and slightly lower than values from wood, indicating a preferential utilization of syringic units relative to vanillic units during tissues decomposition. Results highlighted elevated (Ad/Al) ratios of both vanillic, and syringic phenols that reached 1.37 and 0.38 respectively. Elevated (Ad/Al) ratios usually result from oxidation of phenylpropyl side chain of lignin units (Ertel and Hedges, 1984; Hedges et al., 1988; Prahl et al., 1994). In 1988, Hedges et al. qualified elevated (Ad/Al) $v$ as specific and indelible indicators of lignin degradation in subaerial terrestrial environments. Our observations suggest that propyl side chain oxidation could also happen in saline waterlogged mangrove sediments. The process of lignin decomposition considered here is typical for suboxic conditions. In waterlogged mangrove sediments, these conditions occur thanks to the ability of the radial cable roots of A. geminans to aerate the upper sediment (Scholander et al., 1955), thus resulting in Eh's that can reach values higher than $400 \mathrm{mV}$ (Marchand ef al., 2004).

In sediments collected in the older stages of Avic ennia $(C 12, C 20)$ and in the $\mathrm{R}$. mangle mangrove forests (C25), S/V values slightly varied without any obvious trend, however ratios were always lower than those measured on plants, confirming a preferential utilization of $S$ unit during tissues decomposition. (Ad/Al) ratios of syringic phenols were elevated like in the young forest, thus highlighting side chain oxidation. However, in the layer of freshly introduced OM, (Ad/Al) ratios of vanillic phenols were very low and stable, the opposite to what happened in the young mangrove swamps. These low and stable ratios may result from another process of lignin decomposition that is aromatic ring cleavage. In contrast to phenyl-propyl chain oxidation, aromatic ring cleavage may be the predominant mechanism of lignin degradation under anaerobic conditions (Haddad et al., 1992; Dittmar and Lara, 2001). Lignin was thought to be refractory in anaerobic conditions, mainly because fungi, the most important wood decaying organisms are aerobes. However, Colberg and Young (1982) and 
Benner et al 1984a and Benner et al 1984b demonstrated that lignin can be degraded at a significant rate by bacteria. Then, many works made in different environments, showed that lignin degradation in anaerobic conditions can occur without any changes in the (Ad/Al) of the vanillic unit (Hedges et al., 1985; Benner et al., 1990; Opsahl and Benner, 1995; Dittmar and Lara, 2001). Mangrove sediments beneath older A. geminans swamps were submitted to contrasting redox conditions due to seasonal variability. During the rainy season, low Eh values associated with high sulphur concentrations (Marchand et al., 2004) denoted sulphate-reducing processes similar to those observed under R. mangle stands (C25), except near the cable-root system, where oxidation processes can occur in a way similar to what was observed in young mangroves. In this environment, sulphate reducing conditions are promoted by the available organic matter, stabilization of sediment and waterlogging. Consequently during the rainy season, lignin from hardwood debris might be altered by ring chain cleavage, thus explaining the low and stable values of (Ad/Al) encountered for the vanillic unit. The higher value of this ratio at $14 \mathrm{~cm}$ depth in the $\mathrm{C} 12$ core may denote the proximity of the cable roots. Nevertheless, the vertical shifting of water table during dry seasons led to a suboxic layer of sulphur oxidation, characterized by a really acidic pH (close to 4.5) (Marchand et al., 2004), that may induce propyl side chain oxidation. Consequently beneath the older stages of forest development, sedimentary organic matter was decomposed both during anoxic and suboxic conditions, and evidence of lignin decomposition either of suboxic and anaerobic conditions was found.

\subsubsection{Basal deposits}

In most mangroves, total neutral sugars increased in the basal deposit, beneath $30 \mathrm{~cm}$ deep. For example, in the mature mangrove forest (C20) they increased from $20 \mathrm{mmol} \mathrm{C} / \mathrm{mol}$ TOC at $35 \mathrm{~cm}$ to more than $80 \mathrm{mmol} \mathrm{C} / \mathrm{mol}$ $\mathrm{OC}$ at $80 \mathrm{~cm}$ depth. This increase is mainly due to deoxy sugars (rhamnose and fucose), the amount of which represented more than $50 \%$ of total neutral sugars. As stated in a previous paragraph, such a high deoxy sugar content might be derived from bacteria (Hedges et al., 1988) or from plankton ( $\underline{\text { Hicks }}$ et al., 1994). On the other hand, the high deoxy sugar content was associated with elevated (Ad/Al)v ratios; e.g., in the mature mangrove $(\mathrm{Ad} / \mathrm{Al}) \mathrm{v}$ ratio increased from less than 0.1 in the upper $35 \mathrm{~cm}$ to 1.24 at $80 \mathrm{~cm}$ depth. As noted previously, elevated (Ad/Al)v ratios provide a good indication of lignin degradation in suboxic conditions (Hedges et al., 1988; Goñi et al., 1993). Goñi and Hedges (1992) observed that vanillic ester bound to polysaccharides in soft wood tissues could lead to elevated (Ad/Al)v ratios in non degraded samples. However, at the considered depths, no freshly introduced organic matter was observed. These basal deposits were characterized by constant anaerobic conditions and an organic content mostly made of oxidized allochthonous debris derived from Amazonian detritus, and with very few autochthonous $O M$ inherited from scarce anchorage roots (Marchand et al., 2003). $\mathrm{C} / \mathrm{N}$ ratios were close to 10 and 
${ }^{13} \mathrm{C}$ ranged from -25 to $-28 \%$. Hedges et al. (1986) observed that particulate organic material from the Amazon River was characterized by high $(\mathrm{Ad} / \mathrm{Al})_{v},(>0.8)$, and low $\mathrm{C} / \mathrm{N}$ ratios (close to 10). Both are consistent with the presence of ligneous debris, most probably oxidized during their mobilization towards the river and their transport to the sea. This oxidation process may have continued during sediment migration along the coastline of the Guianas, and as a result of frequent sediment reworking (Aller, 1998). Later, Hedges et al. (1994) reported high deoxy sugars content for the same particulate material. Consistently, we suggest that high deoxy sugar contents and $(\mathrm{Ad} / \mathrm{Al})_{v}$ values at depth, most probably resulted from preferential accumulation of allochthonous oxidized debris from the Amazon watershed.

The high sedimentary organic content at depth (>20 cm deep) in the "recolonized cemetery mangrove" (core C2) derived from an ancient buried root system belonging to the previous generation of mangroves, as demonstrated by the high $\mathrm{C} / \mathrm{N}$ ratios, lower $\delta{ }^{13} \mathrm{C}$ values, and $\mathrm{C} / \mathrm{V}$ ratios close to 0 . At $25 \mathrm{~cm}$ depth, $S / \mathrm{V}$ were low and elevated (Ad/Al)s may denote suboxic decomposition of lignin, as stated previously. These decaying ancient roots were situated in the layer that was aerated by living roots, whereas deeper, the buried roots of the same previous generation were submitted to continuous anoxic conditions. At depth, few evidences of lignin decomposition were observed. S/V ratios were high and (Ad/Al)s were low and stable, displaying values close to those of living roots. The slightly increased $(\mathrm{Ad} / \mathrm{Al})_{v}$, accompanied by an increase in rhamnose may rather result from an increased content in oxidized debris inherited from the Amazonian discharge, than from lignin decomposition. Consequently, on the one hand, these results suggests that in total anoxic conditions few lignin decomposition were observed, whereas on the other hand, the low neutral monosaccharide content suggests than even in such conditions the compounds were efficiently degraded.

\section{Conclusions}

The comparative study of lignin and carbohydrate composition combined with elemental (carbon and nitrogen) and stable isotopic $\left(\delta^{13} \mathrm{C}\right)$ data gave a more refined assessment of organic matter sources and diagenesis during specific stages of mangrove development in French Guiana than that obtained from bulk analyses (Marchand et al., 2003). The main conclusions of this study can be summarized as followed:

1 The proportions of the various neutral carbohydrates present in sediments reflect selective degradation. Cellulosic glucose and total xylose appear to be the two most labile neutral sugars, whereas arabinose, rhamnose, fucose and hemicellulosic glucose highlight in situ microbial syntheses.

2 Debris derived from mangrove roots, which strongly contributed to the enrichment of sediment in OM in this specific environment, are discriminated 
using their content of xylose, and cellulosic monosaccharides and their low $\mathrm{C} / \mathrm{V}$ ratios.

3 Algal mats frequently developing at the sediment surface in young mangrove swamps are most probably responsible for abundant rhamnose. Rhamnose and fucose were most probably introduced at depth by fungi and bacteria that thrived at the expense of vascular plant debris.

4 Carbohydrates are rapidly degraded at the water-sediment interface. Further degradation at depth does not seem to be influenced by variations of the redox conditions.

5 In contrast, lignin derived phenols distribution varied with environmental conditions. Beneath the young mangrove forests, propyl side chain oxidation occur in suboxic conditions due to aeration of the sediment upper layer by $A$. geminans radial cable roots. Within the older swamps, lignin decomposition also occur by ring chain cleavage. In permanent anoxic conditions, few evidences of lignin decomposition are observed.

6 High $(\mathrm{Ad} / \mathrm{Al})_{v}$ ratios combined with high deoxy sugars (fucose and rhamnose) contents in basal deposits witness an accumulation of strongly oxidized debris derived from the Amazon and further oxidized during transport over long distances.

In summary, this study illustrates the various decomposition pathways that can affect lignin and cellulose from vascular plant debris in waterlogged and hypersaline mangrove sediments submitted to variable redox conditions. Decomposition pathways and products depend on such environmental conditions as vegetal species, stages in forest development and seasonal settings.

\section{Acknowledgments}

This research was supported by INSU and the PNEC Guyane. The authors would like to thank IRD Cayenne and F. Fromard (Univ. P. Sabatier, Toulouse, France) for their valuable assistance on field. The help of R.C. Aller (Stony Brook University, USA) and F. Baltzer (Univ. Paris XI, France), for the improvement of this manuscript is gratefully acknowledged. We are also grateful to J. Middelburg (NIOO-KNAW, Netherland), and three anonymous reviewers for constructive

\section{References}

Aller 1998 R.C. Aller, Mobile deltaic and continental shelf muds as suboxic, fluidized bed reactors, Mar. Chem. 61 (1998), pp. 143-155. 
Alongi et al 1999 D.M. Alongi, F. Tirendi, L.A. Trott and G.J. Brunskill, Mineralisation of organic matter in intertidal sediments of a tropical semienclosed delta, Estuar. Coastal Shelf Sc i. 48 (1999), pp. 451-467.

Aspinall 1970 G.O. Aspinall, Polysaccharides, Pergamon Press (1970).

Béguin and Aubert 1994 P. Béguin and J.-P. Aubert, The biological degradation of cellulose, FEMS Mic robiol. Rev. 13 (1994), pp. 25-58.

Benner and Hodson 1985 R. Benner and R.E. Hodson, Microbial degradation of the leachable and lignocellulosic componentes of leaves and wood from Rhizophora mangrove in a tropical mangrove swamp, Mar. Ecol. Prog. Ser. 23 (1985), pp. 221-230.

Benner et al 1990 R. Benner, K. Weliky and J.I. Hedges, Early diagenesis of mangrove leaves in a tropical estuary Molecular-level analyses of neutral sugars and lignin-derived phenols, Geochim. Cosmochim. Acta 54 (1990), pp. 1991-2001.

Benner et al 1984a R. Benner, A.E. Maccubin and R.E. Hodson, Anaerobic biodegradation of the lignin and polysaccharide components of lignocellulose and synthetic lignin by sediment microflora, Appl. Environ. Mic robiol. 47 (1984), pp. 998-1004.

Benner et al 1984b R. Benner, S.Y. Newell, A.E. Maccubin and R.E. Hodson, Relative contributions of bacteria and fungi to rates of degradation of lignocellulosic detritus in salt marsh sediments, Appl. Environ. Microbiol. 48 (1984), pp. 36-40.

Bergamashi et al 1997 B.A. Bergamashi, E. Tsamakis, R.G. Keil, T.I. Eglinton, D.B. Montluçon and J.I. Hedges, The effect of grain size and surface area on organic matter, lignin and carbohydrate concentration and molecular compositions in Peru Margin sediments, Geochim. Cosmochim. Acta $6 \mathbf{1}$ (1997), pp. 1247-1260.

Bianchi et al 1999 T.S. Bianchi, M. Argyrou and H.F. Chippett, Contribution of vascular-plant carbon to surface sediments across the coastal margin of Cyprus (eastern Mediterranean), Org. Geochem. 30 (1999), pp. 287-297.

Bouillon et al 2003 S. Bouillon, F. Dahdouh-Guebas, A.V.V.S. Rao, N. Koedam and F. Dehairs, Sources of organic carbon in mangrove sediments variability and possible ecological implications, Hydrobiol. 495 (2003), pp. 33-39.

Clough 1992 B.F. Clough, Primary productivity and growth of mangrove forests In: A.I. Robertson and D.M. Alongi, Editors, Tropical Mangrove Ecosystem, American Geophysical Union, Washington, DC (1992), pp. 225-249 Coastal and Estuarine Studies $\mathrm{N}^{\circ} 41$. 
Colberg and Young 1982 P.J. Colberg and L.Y. Young, Biodegradation of lignin derived molecules under anaerobic conditions, Can. J. Microbiol. 28 (1982), pp. 886-889.

Cowie and Hedges 1984 G.L. Cowie and J.I. Hedges, Carbohydrate sources in a coastal marine environment, Geochim. Cosmochim. Acta 48 (1984), pp. 2075-2087.

Cowie et al 1992 G.L. Cowie, J.I. Hedges and S.E. Calvert, Sources and reactivities of amino acids, neutral sugars and lignin in an intermittently anoxic marine environment, Geochim. Cosmochim. Acta 56 (1992), pp. 1963-1978.

Dittmar and Lara 2001 T. Dittmar and R.J. Lara, Molecular evidence for lignin degradation in sulfate reducing mangrove sediments (Amazonia, Brazil), Geochim. Cosmochim. Acta 74 (2001), pp. 1417-1428.

Dodd et al 1998 R.S. Dodd, Z.A. Rafii, F. Fromard and F. Blasco, Evolutionary diversity among Atlantic coast mangroves, Acta Oecolog. 19 (1998), pp. 323330.

Ertel and Hedges 1984 J.R. Ertel and J.I. Hedges, The lignin component of humic substances Distribution among soil and sedimentary humic, fulvic and base insoluble fractions, Geochim. Cosmochim. Acta 48 (1984), pp. 20652074.

Ertel and Hedges 1985 J.R. Ertel and J.I. Hedges, Sources of sedimentary humic substances vascular plant debris, Geochim. Cosmochim. Acta 49 (1985), pp. 2097-2107.

Fromard et al 1998 F. Fromard, H. Puig, E. Mougin, G. Marty, J.L. Betoulle and L. Cadamuro, Structure, above ground biomass and dynamics of mangrove ecosystems New data from French Guiana, Oecolog. 115 (1998), pp. 39-53.

Goñi and Hedges 1992 M.A. Goñi and J.I. Hedges, Lignin dimers structures, distribution and potential geochemical applications, Geochim. Cosmochim. Acta 56 (1992), pp. 4025-4043.

Goñi and Hedges 1995 M.A. Goñi and J.I. Hedges, Sources and reactivities of marine-derived organic matter in coastal sediments as determined by alkaline CuO oxidation, Geochim. Cosmochim. Acta 59 (1995), pp. 29652981.

Goñi et al 1993 M.A. Goñi, B.N. Nelson, R.A. Blanchette and J.I. Hedges, Fungal degradation of wood lignins Geochemical perspectives from CUOderived phenolic dimers and monomers, Geochim. Cosmochim. Acta 57 (1993), pp. 3985-4002. 
Greenland and Oades 1975 D.J. Greenland and J.M. Oades, Saccharides In: J.E. Gieseking, Editors, Soil Components, Springer-Verlag (1975), pp. 213-262.

Haddad et al 1992 R.I. Haddad, S.Y. Newel, C.S. Martens and R.D. Fallon, Early diagenesis of lignin-associated phenolics in the salt marsh grass Spartina alterniflora, Geochim. Cosmoc him. Acta 56 (1992), pp. 3751-3764.

Hedges and Ertel 1982 J.I. Hedges and J.R. Ertel, Characterization of lignin by capillary gas chromatography of cupric oxide oxidation products, Anal. Chem. 54 (1982), pp. 1174-1178.

Hedges and Mann 1979a J.I. Hedges and D.C. Mann, The characterization of plant tissues by their lignin oxidation products, Geochim. Cosmochim. Acta $\mathbf{4 3}$ (1979), pp. 1803-1807.

Hedges and Mann 1979b J.I. Hedges and D.C. Mann, The lignin geochemistry of marine sediments from the southern Washington coast, Geochim. Cosmochim. Acta 43 (1979), pp. 1809-1818.

Hedges and Parker 1976 J.I. Hedges and P.R. Parker, Land-derived organic matter in surface sediments from the Gulf of Mexico, Geochim. Cosmochim. Acta 40 (1976), pp. 1019-1029.

Hedges et al 1997 J.I. Hedges, R.G. Keil and R. Benner, What happens to terrestrial organic matter in the ocean?, Org. Geochem. 27 (1997), pp. 195212.

Hedges et al 1988 J.I. Hedges, R.A. Blanchette, K. Weliky and A.H. Devol, Effect of fungal degradation on the $\mathrm{CuO}$ oxidation products of lignin $\mathrm{A}$ controlled laboratory study, Geochim. Cosmochim. Acta $\mathbf{5 2}$ (1988), pp. 27172726 .

Hedges et al 1985 J.I. Hedges, G.L. Cowie, J.R. Ertel, R.J. Barbour and P.G. Hatcher, Degradation of carbohydrates and lignins in buried woods, Geochim. Cosmochim. Acta 49 (1985), pp. 701-711.

Hedges et al 1986 J.I. Hedges, W.A. Clark, P.D. Quay, J.E. Richey, A.H. Devol and U.M. Santos, Composition and fluxes of particulate organic matter in the Amazon River, Limnol. Oceanogr. 31 (1986), pp. 717-738.

Hesse 1961 P.R. Hesse, Some differences between the soils of Rhizophora and Avic ennia mangrove swamp in Sierra Leone, Plant Soil 14 (1961), pp. 335-346.

Hicks et al 1994 R.E. Hicks, C.J. Owen and P. Aas, Deposition, resuspension and decomposition of particulate organic matter in the sediment of Lake Itasca, Minnesota, USA, Hydrobiol. 284 (1994), pp. 79-91. 
Ittekkot 1988 V. Ittekkot, Global trends in the nature of organic matter in river suspensions, Nature 332 (1988), pp. 436-438.

Kerhervé et al 2002 P. Kerhervé, R. Buscail, F. Gadel and L. Serve, Neutral monosaccharides in surface sediments of the northwestern Mediterranean Sea, Org. Geochem. 33 (2002), pp. 421-435.

Lallier-Vergès et al 1998 E. Lallier-Vergès, B.P. Perrussel, J.R. Disnar and F. Baltzer, The relationship between environmental conditions and the diagenetic evolution of organic matter derived from higher plant in a present mangrove swamp system (Guadeloupe, French West Indies), Org. Geochem. 29 (1998), pp. 1663-1686.

Lallier-Vergès et al 1999 E. Lallier-Vergès, J.R. Disnar, O. Maman, O. Sagot, B. Guillet and P. Albéric, Phenols and sugars in hydromorphous soils from Guadeloupe (FWI) relationships with vegetal precursors and degradation conditions, EAOG meeting, Ed. Tübitak, Marama Research Center 2 (1999), pp. 565-566.

Marchand et al 2003 C. Marchand, E. Lallier-Vergès and F. Baltzer, The composition of sedimentary organic matter in relation to the dynamic features of a mangrove-fringed coast in French Guiana, Estuar. Coastal Shelf Sci. 56 (2003), pp. 119-130.

Marchand et al 2004 C. Marchand, F. Baltzer, E. Lallier-Vergès and P. Albéric, Interstitial water chemistry in mangrove sediments in relationship to species composition and developmental stage. (French Guiana), Mar. Geol. 208 (2004), pp. 361-381.

Meybeck 1982 M. Meybeck, Carbon, nitrogen and phosphorus transport by world rivers, Am. J. Sci. 282 (1982), pp. 401-450.

Meyers 1994 P.A. Meyers, Preservation of elemental and isotopic source identification of sedimentary organic matter, Chem. Geol. 144 (1994), pp. 289-302.

Moers et al 1990 M.E.C. Moers, M. Baas, J.W. De Leuw, J.J. Boon and P.A. Schenck, Occurrence and origin of carbohydrates in peat samples from a red mangrove environment as reflected by abundances of neutral monosaccharides, Geochim. Cosmochim. Acta 54 (1990), pp. 2463-2472.

Nierop et al 2001 K.G.J. Nierop, B. van Lagen and P. Buurman, Composition of plant tissues and soil organic matter in the first stages of a vegetation succession, Geoderma 100 (2001), pp. 1-24.

Odum and Heald 1975 W. Odum and E. Heald, The detritus-based food web of an estuarine mangrove community, Estuar. Res. 1 (1975), pp. 265-286. 
Ogier et al 2001 S. Ogier, J.-R. Disnar, P. Albéric and G. Bourdier, Neutral carbohydrate geochemistry of particulate material (trap and core sediments) in an eutrophic lake (Aydat, France), Org. Geochem. 32 (2001), pp. 151-162.

Opsahl and Benner 1995 S. Opsahl and R. Benner, Early diagenesis of vascular plant tissues Lignin and cutin decomposition and biogeochemical implications, Geochim. Cosmochim. Acta 59 (1995), pp. 4889-4904.

Opsahl and Benner 1999 S. Opsahl and R. Benner, Characterization of carbohydrates during early diagenesis of five vascular plant tissues, Org. Geochem. 30 (1999), pp. 83-94.

Prahl et al 1994 F.G. Prahl, J.R. Ertel, M.A. Goñi, M.A. Sparrow and B. Eversmayer, Terrestrial organic carbon contributions to sediments on the Washington margin, Geochim. Cosmochim. Acta 58 (1994), pp. 3035-3048.

Sarkanen and Ludwig 1971 K.V. Sarkanen and C.H. Ludwig, WileyIntersciences, Lignins (1971).

Scholander et al 1955 P.F. Scholander, L. Van dam and S.I. Scholander, Gas exchange in the roots of mangrove, Am. J. Bot. 42 (1955), pp. 92-98

Schlesinger and Melack 1981 W.H. Schlesinger and J.M. Melack, Transport of organic carbon in the world's rivers, Tellus 33 (1981), pp. 172-187.

Spalding et al 1997 M.D. Spalding, F. Blasco and C.D. Field, World Mangrove Atlas, The International Society for Mangrove Ecosystem, Okinawa, Japan (1997), p. 178.

Stevenson 1994 F.J. Stevenson, Humus Chemistry (2nd ed.), John Wiley, New York (1994).

Stout et al 1988 S.A. Stout, J.J. Boon and W. Spackman, Molecular aspects of the peatification and early coalification of angiosperm and gymnosperm woods, Geochim. Cosmochim. Acta 52 (1988), pp. 405-414. 